\title{
dSLo Interacting Protein 1, a Novel Protein That Interacts with Large-Conductance Calcium-Activated Potassium Channels
}

\author{
Xiao-ming Xia, Birgit Hirschberg, Sarah Smolik, Michael Forte, and John P. Adelman \\ Vollum Institute, Oregon Health Sciences University, Portland, Oregon 97201
}

Large-conductance calcium-activated potassium channels (BK channels) are activated by depolarized membrane potential and elevated levels of intracellular calcium. BK channel activity underlies the fast afterhyperpolarization that follows an action potential and attenuates neurotransmitter and hormone secretion. Using a modified two-hybrid approach, the interaction trap, we have identified a novel protein from Drosophila, dSLIP1 (dSLo interacting protein), which specifically interacts with Drosophila and human BK channels and has partial homology to the PDZ domain of $\alpha 1$ syntrophin. The dSLIP1 and dSlo mRNAs are expressed coincidently throughout the Drosophila nervous system, the two proteins interact in vitro, and they may be coimmunoprecipitated from transfected cells. Coexpression of dSLIP1 with dSlo or hSlo BK channels in Xenopus oocytes results in reduced currents as compared with expression of BK channels alone; current amplitudes may be rescued by coexpression with the channel domain that interacts with dSLIP1. Single-channel recordings and immunostaining of transfected tissue culture cells suggest that dSLIP1 selectively reduces Slo BK currents by reducing the number of BK channels in the plasma membrane.

Key words: BK channels; two-hybrid; interacting protein; chaperone protein; regulated expression; current density
Neuronal action potentials are followed by an afterhyperpolarization (AHP) that has several kinetic components and may have profound consequences for the firing pattern of the neuron. During an action potential the concerted influences of increased intracellular calcium and depolarized membrane potential activate two classes of calcium-activated potassium channels: largeconductance calcium- and voltage-dependent channels (BK channels) and small-conductance (SK) channels activated only by calcium. Together, these distinct classes of calcium-activated potassium channels are responsible for the different kinetic components of the AHP. Application of BK channel blockers such as charybdotoxin (CTX) or tetraethylammonium (TEA) has shown that $\mathrm{BK}$ channels contribute to action potential repolarization and underlie the fast component of the AHP (fAHP), which develops rapidly (rise time 1-2 msec) and decays within tens of milliseconds (Lancaster and Nicoll, 1987; Storm, 1987), whereas the subsequent slow components (sAHP) are attributable to SK channels and underlie spike frequency adaptation (Hotson and Prince, 1980; Madison and Nicoll, 1984; Yarom et al., 1985).

Regulation of BK channel activity exerts a powerful modulation on neuronal excitability. Electrophysiological studies have shown that native BK channels are regulated by a wide range of second messengers, including several protein kinases and protein phosphatases (Ewald et al., 1985; Chung et al., 1991; Reinhart et al., 1991) and G-proteins (Cole and Sanders, 1989; Toro et al., 1990; Scornik et al., 1992). In addition, mammalian BK channels have a closely associated $\beta$-subunit that modifies the calcium

\footnotetext{
Received Nov. 3, 1997; revised Jan. 12, 1998; accepted Jan. 15, 1998.
}

This work was supported by National Institutes of Health grants to J.P.A. We thank our colleagues Jim Maylie, Chris Bond, Ian Roberts, Bill Wolfgang, and Stan Hollenberg for fruitful discussions. We also thank Erica Golemis and Roger Brent for supplying interaction trap reagents before their publication and for continued technical advice during the course of these experiments.

Correspondence should be addressed to Dr. John P. Adelman, Vollum Institute, Oregon Health Sciences University, Portland, OR 97201.

Copyright (C) 1998 Society for Neuroscience $0270-6474 / 98 / 182360-10 \$ 05.00 / 0$ sensitivity of the channel and that itself may be the target for regulatory second messengers (McManus et al., 1995; Dworetzky et al., 1996; Hanner et al., 1997).

Other mechanisms may influence the fAHP via indirect effects on BK channels. There is evidence that, at least in some neuronal cell types, BK and voltage-dependent calcium channels (VDCCs) are associated closely and may be coupled physically (Gola and Crest, 1993; Robitaille et al., 1993; Issa and Hudspeth, 1994). Other post-translational modulatory effects on BK channels have been described, but the underlying molecular mechanisms have not yet been established (Subramony et al., 1996; Subramony and Dryer, 1997). For other voltage-gated potassium channels, a distinct $\beta$-subunit, $\operatorname{Kv} \beta 2$, associates with the $\alpha$-subunits early in channel biosynthesis and exerts dramatic, chaperone-like effects on the $\alpha$-subunits, including stabilization and increased cell surface expression (Rettig et al., 1994; Shi et al., 1996). These results suggest that the subcellular distribution and density of BK channels will affect the kinetics of the fAHP and neuronal excitability.

Both classes of calcium-activated potassium channels now have been cloned. Heterologous expression studies have demonstrated that the cloned channels faithfully reproduce the biophysical characteristics of their native counterparts (Atkinson et al., 1991; Adelman et al., 1992; Butler, 1993; Köhler et al., 1996). To identify other proteins, such as $\beta$-subunits, that interact with BK channels and influence BK expression, we used the C-terminal domain of a cloned BK channel in a two-hybrid screen. One of the clones identified as interacting with the dSlo BK channel, dSLIP1 (dSLo interacting protein), appears to regulate the number of BK channels in the plasma membrane.

\section{MATERIALS AND METHODS}

Interaction trap. A detailed description of the interaction trap has been presented previously (Gyuris et al., 1993). Briefly, the C-terminal 499 residues of dSlo (dSlo-C665-1164) were fused to the C-terminal oligomerization region of LexA in the parent plasmid pEG202, which has HIS3 as a selectable marker and a $2 \mu \mathrm{m}$ replicator. In this plasmid the 
LexA-dSlo-C665-1164 fusion (the "bait") is expressed constitutively from the yeast ADH promoter/terminator. The bait plasmid was transformed into the yeast strain EGY195, auxotrophic for histidine (his3), tryptophan (trp1), uracil (ura3-52), and leucine (leu2). For selection of interacting proteins, EGY195 contains an integrated copy of the leu2 gene in which the upstream regulatory sequences have been replaced by two LexA operators. EGY195 also carries pSH18-34, which contains an expression cassette for $\beta$-galactosidase driven by the Gal1 promoter under the control of four LexA operators, as well as the URA3 selectable marker. The presence of this plasmid allows an independent verification of LexA-driven transcriptional activation. EGY195 containing the bait plasmid and the $\beta$-galactosidase reporter plasmid was transformed with a cDNA library constructed in pJG4-5. Expression of the cDNA is under the control of the Gal1 promoter, and the expressed protein is a fusion with (N- to $\mathrm{C}-$ ) a nuclear localization signal, the B42 transcriptional activation domain (Ma and Ptashne, 1988), the HA1 epitope tag, the cDNA, and the ADH transcriptional terminator. pJG4 -5 also carries a $\mathrm{TRP}^{+}{ }^{+}$selectable marker and a $2 \mu \mathrm{m}$ replicator. Thus, a cell that has been transformed with a cDNA plasmid encoding a protein that interacts with the bait will activate transcription of leu 2 and $\beta$-galactosidase independently via LexA-LexA operator interactions, when grown on galactose as the carbon source; selection is for his, trp, ura, and leu prototrophy, and colonies are blue in a $\beta$-galactosidase filter assay.

Yeast containing the bait plasmid and the $\beta$-galactosidase reporter plasmid were transformed with a Drosophila embryo cDNA library (generous gift of Dr. Russ Finley, Harvard University, Cambridge, MA) according to a variation of the procedure of Schiestl and Gietz (1989). The transformation mix was plated to galactose $\mathrm{Ura}^{-}, \mathrm{Trp}^{-}, \mathrm{His}^{-}$, and Leu ${ }^{-}$plates; transformation complexity $\left(\sim 1.5 \times 10^{6}\right)$ was determined by plating an aliquot to this medium containing leucine. After $4 \mathrm{~d}, \sim 760$ $\mathrm{LEU}^{+}$colonies appeared. These were patched to glucose Ura ${ }^{-}, \operatorname{Trp}^{-}$, and $\mathrm{His}^{-}$plates and scored on galactose $\mathrm{Ura}^{-}, \mathrm{Trp}^{-}, \mathrm{His}^{-}$, and $\mathrm{Leu}^{-}$ plates and a $\beta$-galactosidase filter assay, yielding eight clones that grew strongly in the absence of leucine and that also were deep blue on X-gal. These plasmids were rescued by transforming yeast miniprep DNA into Escherichia coli $\mathrm{KC} 8$, a trp ${ }^{-}$strain, permitting specific rescue of the $\operatorname{trp}^{+}$ prey plasmid. Miniprep DNAs from individual E. coli transformants were retested individually by transforming them back into EGY195 carrying the bait and $\beta$-galactosidase reporter plasmids, as well as into EGY195 carrying only the reporter plasmid. One of the clones was able to induce leucine prototrophy in the absence of the bait plasmid and was eliminated from further analysis. The nucleotide sequences for the other seven clones were determined; three clones, one an RNA binding protein, one a mitochondrial enzyme, and one a transcription factor, were not studied further. The remaining four clones were examined for overlapping expression patterns with dSlo by using in situ hybridization; one clone, dSLIP1, was chosen for further study. EGY195 and pSH18-34 were the generous gifts of Drs. Erica Golemis and Roger Brent (Harvard University, Cambridge, MA). To isolate dSLIP1 5' coding sequences, we performed 5' rapid amplification of cDNA ends (RACE) reactions, as previously described (Bond et al., 1994). Nucleotide sequences were determined by Sequenase (United States Biochemical, Cleveland, $\mathrm{OH}$ ); nucleic acid and protein sequence analyses were performed by using the Genetics Computer Group suite of software (Madison, WI).

Antibodies. Fragments of dSlo (C665-1164 and full-length dSLIP1) were cloned into the polyHis fusion vector pET16 and transformed into E. coli strain BL21(DE3). Fusion proteins were induced by treating the cultures with isopropyl thiogalactoside (IPTG). After induction, bacteria were pelleted and then lysed with lysozyme and sonication. Inclusion bodies were pelleted, washed once with PBS, repelleted, and resuspended in PBS. Inclusion bodies were used to immunize rabbits (Biodesign International, Kennebunkport, ME), and bleeds were assessed individually by probing Western blots of appropriate glutathione $S$-transferase (GST) fusion proteins.

Western blot. For Western blots, Drosophila larvae ( $\sim 1 \mathrm{gm} ; 0-24 \mathrm{hr})$ were homogenized thoroughly and sonicated in $500 \mu$ of $2 \times$ SDS-PAGE loading buffer and pelleted in a table-top centrifuge; $15 \mu \mathrm{l}$ of the supernatant was subjected to $10 \%$ SDS-PAGE. After electrophoresis, proteins were electroblotted to a nitrocellulose filter. The blot was preabsorbed with $2.5 \%$ dry milk in PBS and $0.1 \%$ Triton X-100 and probed at room temperature for $2 \mathrm{hr}$ with a 1:5000 dilution of either dSlo or dSLIP1 antiserum ( $0.5 \%$ dry milk in PBS and $0.1 \%$ Triton X-100). Then the blot was washed three to five times with PBS and $0.1 \%$ Triton $\mathrm{X}-100$, incubated at room temperature for $1 \mathrm{hr}$ with secondary antibody (1:5000 dilution of HRP-conjugated anti-rabbit antibody; Santa Cruz
Biotechnology, Santa Cruz, CA), and washed three to five times with PBS and $0.1 \%$ Triton X-100. ECL detection reagent (Amersham, Arlington Heights, IL) was added and incubated for $1 \mathrm{~min}$ before exposure to x-ray film for $30 \mathrm{~min}$. Finally, the image was scanned by an ARCUS II AGFA scanner (AGFA, Mortel, Belgium).

In situ hybridization to polytene chromosomes and embryo sections. Genome mapping by in situ hybridization to polytene chromosomes was done as described (Quan et al., 1993). Digoxigenin-11-UTP-labeled riboprobes for in situ hybridization to embryos were generated from dSLIP1 and dSlo cDNA clones by in vitro transcription. Subsequent processing of embryos was done as described (O'Neil and Bier, 1994).

Northern blot analysis. Drosophila embryos ( 1 gm; 0-24 hr) were homogenized with a Dounce homogenizer, and poly $\left(\mathrm{A}^{+}\right)$mRNA was oligo-dT-selected; $3 \mu \mathrm{g}$ was prepared as a Northern blot and probed with radiolabeled full-length dSLIP1 antisense RNA (50\% formamide, 5\%

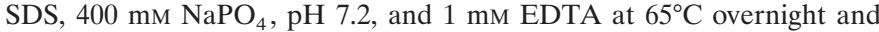
then washed in $1 \% \mathrm{SDS}$ and $0.5 \times \mathrm{SSC}$ at $50^{\circ} \mathrm{C}$ ). The blot was exposed to $\mathrm{x}$-ray film for $18 \mathrm{hr}$ and scanned as described above.

GST pull-down experiments. The indicated fragments of either dSlo or dSLIP1 were fused to GST in pGEX-KG (Pharmacia, Piscataway, NJ) and expressed in E. coli $\mathrm{DH} 5 \alpha$ or were fused to polyHis in pET33b and grown in BL21(DE3) or NovaBlue (DE3; Novagen, Madison, WI). After IPTG induction, bacteria were lysed, and inclusion bodies were solubilized in (in mM) 10 Tris, $\mathrm{pH} 8.0,150 \mathrm{NaCl}, 1$ EGTA, 5 DTT, and 0.2 PMSF with $1.5 \%$ Sarkosyl. GST fusion proteins $(\sim 10 \mu \mathrm{g})$ were batchbound to glutathione-agarose beads (Sigma, St. Louis, MO) in this same buffer, rocked at $4^{\circ} \mathrm{C}$ overnight, and washed five times with PBS. Of the glutathione-agarose beads, $15 \mu \mathrm{l}$ was combined with solubilized His-tag fusion proteins $(\sim 100 \mu \mathrm{g})$ into $1 \mathrm{ml}$ of binding buffer [ containing (in mM) 10 HEPES, pH 7.5, 0.5 DTT, 0.5 EDTA, $150 \mathrm{NaCl}$, and $0.2 \mathrm{PMSF}$ with $0.1 \% \mathrm{NP}-40$ and $5 \mathrm{mg} / \mathrm{ml} \mathrm{BSA}$ ], incubated for $12 \mathrm{hr}$ at $4^{\circ} \mathrm{C}$, and washed three to five times with excess binding buffer; bound proteins were batch-eluted with $30 \mu \mathrm{l}$ of reduced glutathione (Sigma). Eluted proteins $(10 \mu \mathrm{l})$ were mixed with $2 \times$ loading buffer and subjected to $8 \%$ SDS-PAGE. The gel was prepared as a Western blot and probed with either dSlo or dSLIP1 antiserum, as described above.

Coimmunoprecipitations. COS-7 cells were transiently transfected by using calcium phosphate; $48 \mathrm{hr}$ after transfection the cells were harvested and lysed in $50 \mathrm{~mm}$ HEPES, pH 7.5, $100 \mathrm{~mm} \mathrm{NaCl}, 10 \%$ glycerol, $1 \%$ Triton X-100, and 0.2 mM PMSF. After sonication, insoluble debris was removed by a brief centrifugation, and the supernatant was incubated with preimmune serum $(5 \mu \mathrm{l})$ for $1 \mathrm{hr}$ at $4^{\circ} \mathrm{C}$. Protein A-Sepharose CL-4B (Pharmacia) was added and incubated for an additional $3 \mathrm{hr}$ at $4^{\circ} \mathrm{C}$ before centrifugation in a table-top centrifuge. The supernatant was used for coimmunoprecipitations by incubation with $3 \mu \mathrm{l}$ of dSlo antiserum at $4^{\circ} \mathrm{C}$ for $1 \mathrm{hr}$, after which $30 \mu \mathrm{l}$ of protein A-Sepharose CL-4B was added; the mixture was rocked overnight at $4^{\circ} \mathrm{C}$. After centrifugation, the immunoprecipitate was washed extensively with $50 \mathrm{~mm}$ HEPES, $\mathrm{pH} 7.5,100 \mathrm{~mm} \mathrm{NaCl}, 10 \%$ glycerol, $0.1 \%$ Triton X-100, and $0.2 \mathrm{~mm}$ PMSF. Antibody was eluted by the addition of $50 \mu \mathrm{l}$ of $0.1 \mathrm{M}$ glycine, $\mathrm{pH}$ 2.9 , and an aliquot was combined with $2 \times$ loading buffer and subjected to $8 \%$ SDS-PAGE. The gel was prepared as a Western blot and probed with the indicated antiserum, as described above.

Immunocytochemistry. COS-7 cells were plated onto microscope slide coverslips and transiently transfected, using calcium phosphate, with dSlo or dSlo and dSLIP1, each subcloned in pcDNA3 (Invitrogen, San Diego, CA). Forty-eight hours after transfection, cells were washed three times with PBS, then fixed with $4 \%$ paraformaldehyde (for $15 \mathrm{~min}$ at room temperature), washed three times with $0.1 \%$ Triton X-100 in PBS, and washed twice with the same solution with $0.2 \%$ BSA. Cells were preblocked with $10 \%$ horse serum and $0.1 \%$ Triton X-100 in PBS at $4{ }^{\circ} \mathrm{C}$ for $3 \mathrm{hr}$, washed once with $0.1 \%$ Triton X-100 in PBS, and incubated overnight at $4^{\circ} \mathrm{C}$ with rabbit anti-dSlo antiserum (1:1000) in $0.1 \%$ Triton $\mathrm{X}-100$ in PBS. The next morning, cells were washed twice with $0.1 \%$ Triton X-100 in PBS and incubated for $2 \mathrm{hr}$ with biotinylated goat anti-rabbit secondary antibody (1:500; Vector Laboratories, Burlingame, CA), then washed two times with $0.1 \%$ Triton X-100 in PBS, incubated with FITC avidin D (1:200; Vector Laboratories), and finally washed five times with PBS. Coverslips were mounted on slides, and immunostaining was visualized with a fluorescence microscope (Leitz Dialux 22EB, Wetzlar, Germany).

Electrophysiology. In vitro mRNA synthesis and oocyte injections were performed as previously described (Adelman et al., 1992). dSlo was expressed from $\mathrm{pS}^{-}$(A1E1G3; Adelman et al., 1992), dSLIP1 and dSlo-C665-1164 from pBF (generous gift of Dr. Bernd Fakler, University 
A

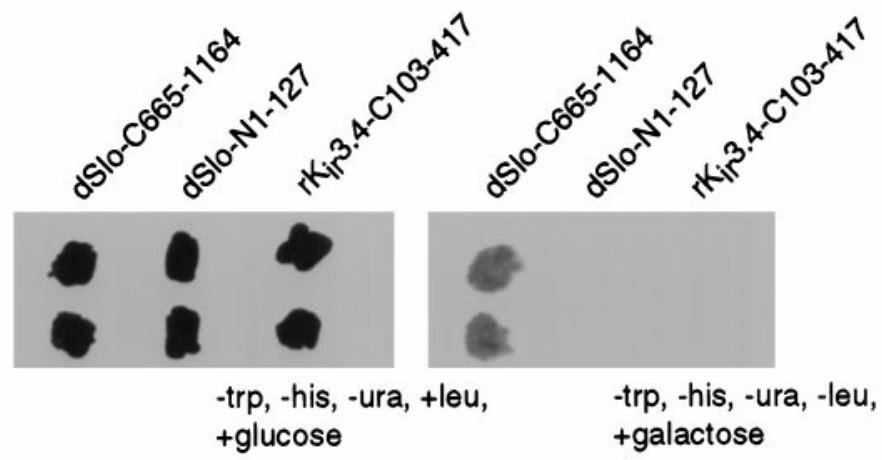

B

001 MSIADVEYEY VVIKINOYDI SHLSRYEAVQ KFLQSKETLV VEIRRQKHNA

051 LDLELKHOSN AKISKVDNPG ELSVLTDKSA EGTITAASAS QQINCPSSTS

101 LKEIETKTPV VLTLRARSHE DRLGSLQAAS KETQTQSVVO TDVLKDNDLV

151 NTITDNFIMH EHHLFEQCLE PEIDIEEVTL VKOVEQSSSN QIOLIVTSSO

201 IQQSSTDTNK ODILGNAAAP GEEVDNSSSA YNTGDSNNSA SPHQNTTNPD

251 EAIATGRKLD * STVIDSPNDH LDATOVSTML LLPFOKSGRI GLCSSNLPTA

Figure 1. $A$, Interaction trap selection for dSLIP1. dSLIP1 was introduced into EGY195 containing the indicated bait plasmids. The plate on the left shows that in the presence of glucose and leucine all combinations permit growth, whereas the plate on the right shows that only the combination of dSLIP1 with dSlo-C665-1164 survives in the presence of galactose and the absence of leucine. dSlo-C665-1164, the C-terminal 499 amino acids of dSlo; $d$ Slo-N1-127, the N-terminal 127 amino acids of dSlo; $r K_{\mathrm{ir}} 3.4-C 103-417$, the C-terminal 314 amino acids of $\mathrm{K}_{\mathrm{ir}} 3.4$ (Krapivinsky et al., 1995a,b). B, Full-length coding sequence of dSLIP1. The domain recovered in the original interaction trap screen started from amino acid 101 and extended through the coding region; potential substrate sites for serine/threonine protein kinases are denoted by asterisks. The domain of dSLIP1 with homology to the PDZ domain of $\alpha 1$ syntrophin is overlined. $C$, The dSLIP1 cDNA encodes the full-length protein. Bacterially expressed dSLIP1 (His-tag fusion protein; lane 1), in vitro translated dSLIP1 (lane 2), and Drosophila embryo proteins (lane 3) were prepared as a Western blot and probed with a polyclonal antiserum directed against recombinant dSLIP1. The dSLIP1 antiserum detected bands of similar molecular weights.

301 YVSERYTNVO SENEIHPLKS DIEILRVKPT DDSYSHCPQF NAPNLSSYHF

351 VSSQEVANRC HISTSLQKNA TLLNGESAEE IPMVWKVRRR PDGTRT of Tübingen, Tübingen, Germany), and the noninactivating version of Shaker from pSK (generous gift of Dr. Ligia Toro, University of California at Los Angeles, Los Angeles, CA). Xenopus care and handling were in accordance with the highest standards of institutional guidelines. Frogs underwent no more than two surgeries, separated by at least three weeks, and surgeries were performed by well established techniques. Frogs were anesthetized with an aerated solution of 3-aminobenzoic acid ethyl ester. Oocytes were studied 3-7 d after injection. Whole-cell currents were measured by a two-electrode voltage clamp with a Geneclamp 500 amplifier (Axon Instruments, Foster City, CA) interfaced to a Macintosh Quadra 800 computer. Data were acquired via Pulse
(HEKA Elektronik, Lambrecht, Germany) at $500 \mathrm{~Hz}$. During recording, oocytes were superfused continuously with ND96 solution containing (in mM) $96 \mathrm{NaCl}, 2 \mathrm{KCl}, 1.8 \mathrm{CaCl}_{2}, 1 \mathrm{MgCl}_{2}$, and 5 HEPES, pH 7.5 with $\mathrm{NaOH}$, at room temperature.

Inside-out macropatches were excised into an intracellular solution containing (in $\mathrm{mm}$ ) $116 \mathrm{~K}$-gluconate, $4 \mathrm{KCl}$, and $10 \mathrm{HEPES}, \mathrm{pH} 7.25$, adjusted with $\mathrm{KOH}$ supplemented with $\mathrm{CaCl}_{2}$ or EGTA or both. To obtain nominally $\mathrm{Ca}$-free solution, we added $1 \mathrm{~mm}$ EGTA. Alternatively, $\mathrm{CaCl}_{2}$ was added to the cytoplasmic solution to give free calcium concentrations of $10-100 \mu \mathrm{M}$. In this case the proportion of calcium binding to gluconate was determined by a computer program ( $\mathrm{CaBuf})$, assuming 

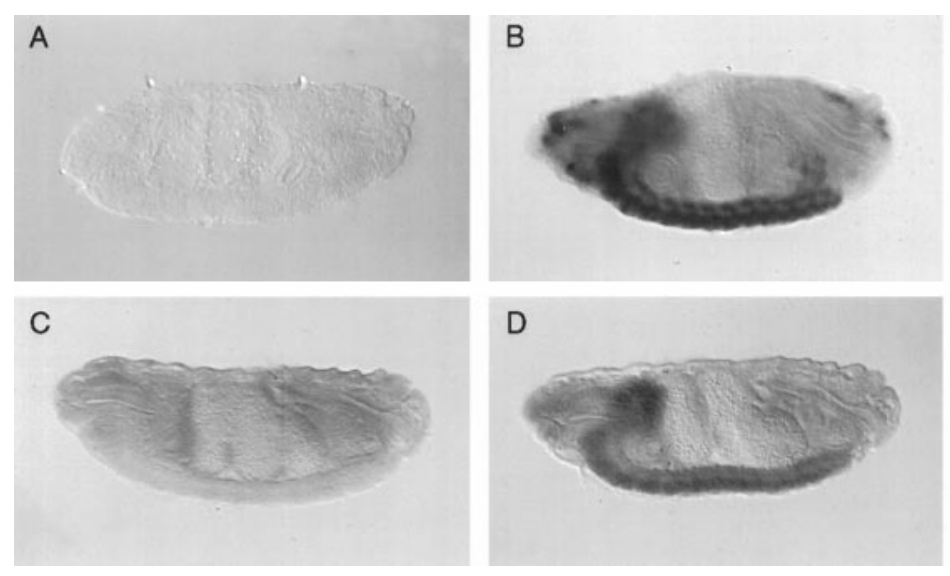
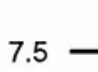

4.4

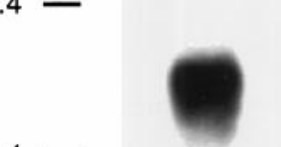

$2.4-$

$1.4-$
Figure 2. Left, dSlo and dSLIP1 mRNAs are expressed in a coincident pattern throughout the Drosophila CNS. Lateral views of whole-mount in situ hybridization in late-stage embryos of sense $(A, C)$ and antisense $(B, D)$ digoxigeninlabeled riboprobes generated from $d s l o$ $(A, B)$ and dSLIP1 $(C, D)$ cDNAs. Strong hybridization of both antisense probes is present in the brain and ventral ganglion. In contrast to $d s l o$, hybridization of dSLIP1 is not present in anterior sensory cells. Anterior, left; posterior, right. Right, Northern blot analysis of dSLIP1 mRNA. Poly $\left(\mathrm{A}^{+}\right)$mRNA $(3 \mu \mathrm{g})$ extracted from Drosophila embryos was prepared as a Northern blot and probed with a full-length radiolabeled dSLIP1 riboprobe; detected was a single band of $\sim 3.6 \mathrm{~kb}$.

A

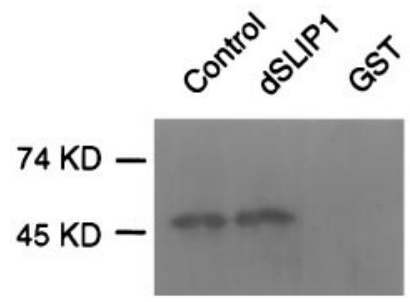

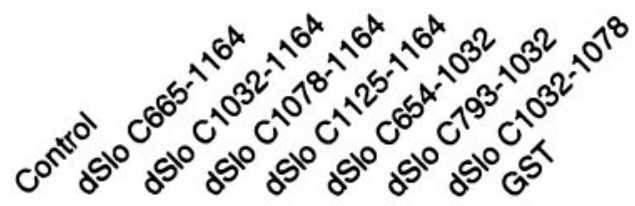

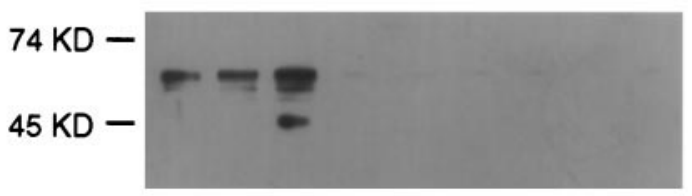

B

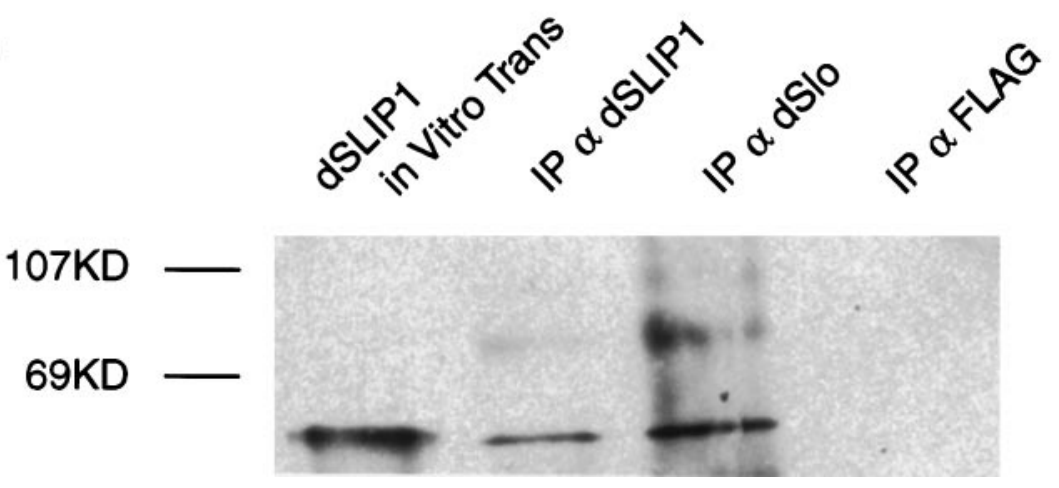

Figure 3. GST pull-down experiments show the interaction between dSlo and dSLIP1. GST fusion proteins, indicated above the blots, were bound to glutathioneagarose beads, and pHis-dSlo (top, left) or pHis-dSLIP1 (top, right) protein was applied. After several washes, retained proteins were eluted with reduced glutathione and prepared as a Western blot. A, Left, Western blot probed with dSlo antibodies. Lane 1 (Control), Bacterially expressed pHis-dSlo-C665-1164 protein, as marker. pHis-dSlo-C665-1164 protein was retained by GST-dSLIP1 (lane 2), but not by GST alone (lane 3). Right, Western blot probed with dSLIP1 antibodies. Lane 1, Bacterially expressed pHis-dSLIP1 alone as marker; lanes 2-9, pHis-dSLIP1 was retained by GST-dSlo-C654-1164 or GST-dSlo-C1032-1164, but not by other C-terminal fragments of dSlo or by GST alone. $B$, Antibodies to dSlo coimmunoprecipitate dSLIP1 from cotransfected COS-7 cells. Western blot was probed with dSLIP1 antibodies. Lane 1, In vitro translated dSLIP1; lane 2, proteins immunoprecipitated with dSLIP1 antibodies; lane 3, proteins immunoprecipitated with dSlo antibodies; lane 4, proteins immunoprecipitated with FLAG antibodies. a stability constant for $\mathrm{Ca}^{2+}$ gluconate of $15.9 \mathrm{M}^{-1}$ (Dawson et al., 1969). Electrodes were pulled from thin-walled filamented borosilicate glass (World Precision Instruments, Sarasota, FL) and filled with (in mM) 116 $\mathrm{K}$-gluconate, $4 \mathrm{KCl}$, and $10 \mathrm{HEPES}, \mathrm{pH}$ 7.25. Electrode resistance was typically $2-5 \mathrm{M} \Omega$. Membrane patches were voltage-clamped by an Axopatch 200A amplifier (Axon Instruments). The data were low-pass Bessel-filtered at $1 \mathrm{kHz}$ and acquired with Pulse software (HEKA Elektronik). Analysis was performed by Pulse and Kaleidagraph (Abelbeck, Reading, PA) software. Macropatch currents were measured during $500 \mathrm{msec}$ voltage steps from a holding potential of $0 \mathrm{mV}$. All experiments were performed at room temperature.
To examine single-channel properties, we used the same solutions as those for macropatch recordings. Electrodes were pulled from Corning 7052 glass (Garner) and had resistances of 9-13 M 2 . Data were filtered at $1 \mathrm{kHz}$ (Bessel), acquired at $10 \mathrm{kHz}$ by using Pulse (HEKA Elektronik), and stored directly on a Macintosh Quadra 650. Recordings were analyzed by MacTac (Bruxton, Seattle, WA). The " $50 \%$ threshold" technique was used to detect openings that were inspected visually and adjusted for their amplitude before being accepted. Amplitude histograms were constructed by using MacTacfit (Bruxton) and fit by a single gaussian distribution. $N P_{\mathrm{o}}$, the product of the single-channel open probability multiplied by the number of channels, was calculated as the sum of 
Table 1. Regions of interaction between dSlo and dSLIP1

Constructs

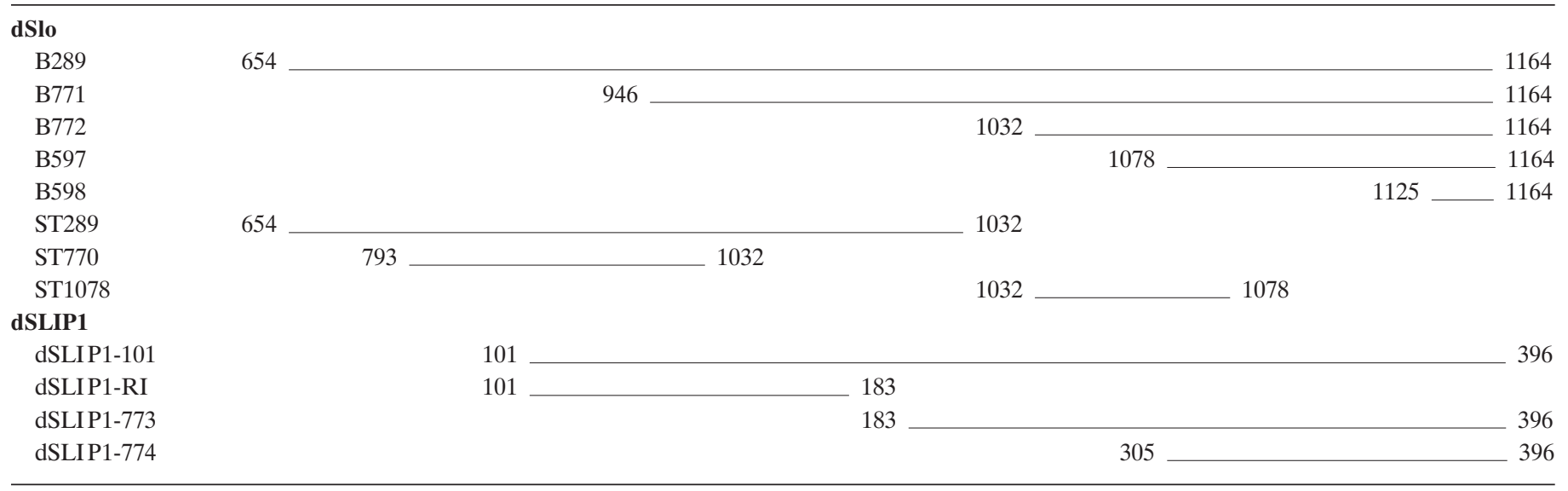

Shown is a diagramatic representation of the regions from dSlo and dSLIP1, which were tested for complementation in the interaction trap.

\begin{tabular}{|c|c|c|c|c|c|c|c|c|}
\hline & \multicolumn{2}{|c|}{ dSLIP1-101 } & \multicolumn{2}{|c|}{$\underline{\mathrm{dSLIP1} 1-\mathrm{RI}}$} & \multicolumn{2}{|c|}{ dSLIP1-773 } & \multicolumn{2}{|c|}{$\underline{\text { dSLIP1-774 }}$} \\
\hline & Growth & $\beta$-gal & Growth & $\beta$-gal & Growth & $\beta$-gal & Growth & $\beta$-gal \\
\hline B289 & +++ & Blue & - & & +++ & Blue & +++ & Blue \\
\hline B771 & +++ & Blue & - & & +++ & Blue & +++ & Blue \\
\hline B772 & +++ & Blue & - & & +++ & Blue & +++ & Blue \\
\hline B597 & + & Blue & \pm & & + & Blue & + & Blue \\
\hline B598 & + & Blue & \pm & & + & Blue & + & Blue \\
\hline ST289 & \pm & & \pm & & \pm & & \pm & \\
\hline ST770 & \pm & & \pm & & \pm & & \pm & \\
\hline ST1078 & \pm & & \pm & & \pm & & \pm & \\
\hline
\end{tabular}

Shown are assay results for growth and $\beta$-galactosidase activity.

the dwell time $\times$ level number divided by the total time. To calculate $P_{\mathrm{o}}$, we estimated $N$ as the maximum number of simultaneously open channels at $100 \mathrm{mV}$ in $10 \mu \mathrm{M}$ calcium.

\section{RESULTS}

\section{Isolation of dSLIP1}

The sequence encoding the C-terminal 500 amino acids of dSlo, a region that does not vary by alternative exon choices among the known dSlo splice variants (Atkinson et al., 1991; Adelman et al., 1992), was used in an interaction trap (Gyuris et al., 1993) screen of a Drosophila embryo cDNA library. From $\sim 10^{6}$ transformed cDNAs, several hundred colonies were obtained on media lacking leucine. These were assayed individually on galactose media for growth in the absence of leucine and $\beta$-galactosidase activity, which eliminated all but eight of the clones. The remaining candidates were rescued, and the nucleotide sequences of the inserts were determined. Four of the clones were discarded; one encoded a RNA binding protein, one encoded a transcription factor, and two contained mitochondrial DNA sequences. The remaining four clones fulfilled the interaction trap requirements for interactions with the C-terminal domain of dSlo, and one clone, dSLIP1 (dSLo interacting protein), was chosen for further study (Fig. $1 A$; see Materials and Methods).

The original dSLIP1 clone contained an open reading frame fused to the B42 transcriptional activation domain, a stop codon, $3^{\prime}$ untranslated sequences, and a poly $\left(\mathrm{A}^{+}\right)$tail. However, this clone did not contain an initiator methionine. To isolate a full- length coding sequence, we performed 5' RACE reactions by using Drosophila head cDNA (Bond, 1994). Analysis of the RACE products extended the N-terminal coding sequence and identified a putative initiator codon. The full-length coding sequence of dSLIP1 predicts a protein of 396 amino acids with five potential substrate sequences for serine/threonine protein kinases (R/KXXT/S; Fig. 1B). Hydropathy analysis did not identify hydrophobic domains that may span the membrane, suggesting that dSLIP1 is a cytoplasmic protein. The dSLIP1 sequence does not show overall homology to any other known protein, but the $\mathrm{N}$ terminus contains a region with homology to the PDZ domain of $\alpha 1$ syntrophin (Fig. 1B; Gibson et al., 1994; Adams et al., 1995). To confirm that the clone encoding dSLIP1 contained the fulllength coding sequence, we prepared bacterially expressed dSLIP1, in vitro translated dSLIP1, and extracts from whole Drosophila embryos as a Western blot. Antisera specific for dSLIP1 recognized bands of the same size (Fig. 1C) only in these samples.

\section{dSlo and dSLIP1 mRNAs are coexpressed in Drosophila}

The expression patterns of dSlo and dSLIP1 mRNAs were compared by using in situ hybridization on embryo whole mounts. dSlo mRNA is heavily expressed throughout the CNS as well as in several peripheral locations (Fig. 2, left, $A, B$ ). dSLIP1 mRNA also is expressed throughout the CNS (Fig. 2, left, $C, D$ ); dSlo and dSLIP1 mRNAs are expressed coincidently in virtually all CNS 
A

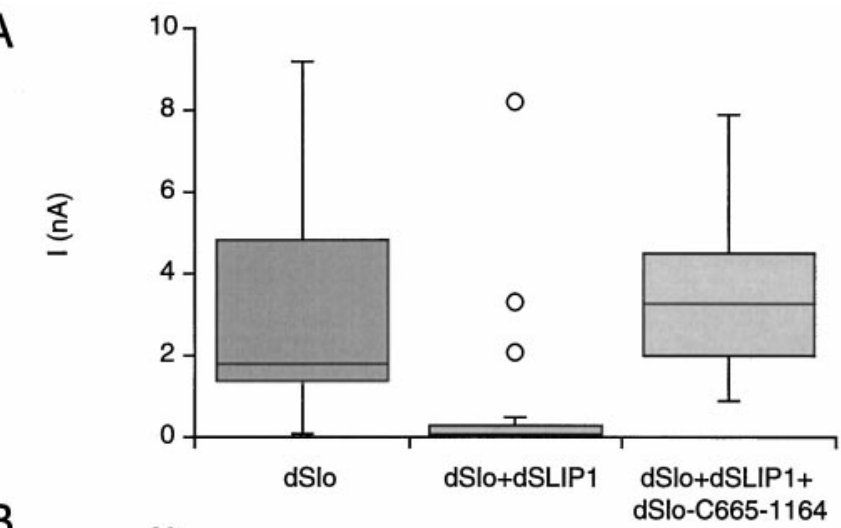

B

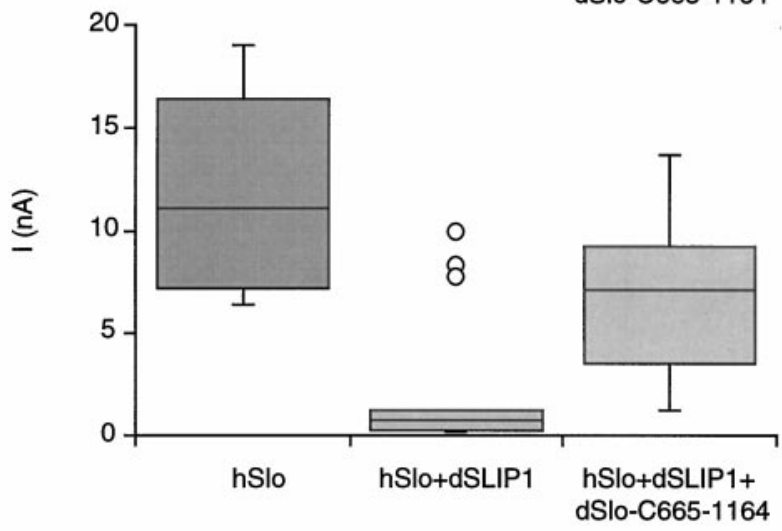

C

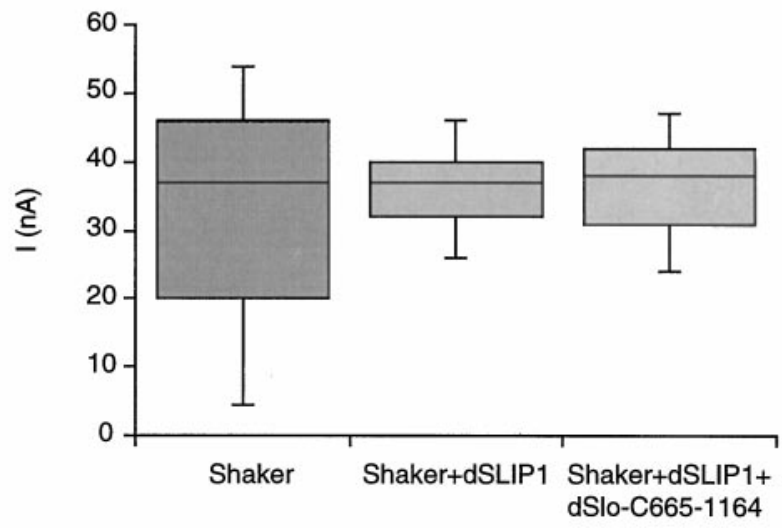

Figure 4. Coexpression with dSLIP1 reduces BK current amplitudes. Current amplitudes at $100 \mathrm{mV}$ were determined in inside-out patches from oocytes excised into $10 \mu \mathrm{M} \mathrm{Ca}^{2+}(A, B)$ or by using the twoelectrode voltage clamp $(C)$. Oocytes expressed dSlo $(A)$, hSlo $(B)$, or Shaker $(C)$ channels either alone (left columns) or in combination with dSLIP1 (middle columns) or with dSLIP1 and dSlo-C665-1164 (right columns). Patch currents are shown as box plots in which the median is represented by a line separating the upper and lower quartiles (UQ, LQ). The box (interquartile distance, IQD) contains $\pm 25 \%$ of the data points; the error bars mark the minimum and maximum values that fall within $\mathrm{UQ}+1.5 \times \mathrm{IQD}$ and $\mathrm{LQ}+1.5 \times \mathrm{IQD}$. The outliers in $A$ and $B$ were recorded from a single oocyte in each case. The numbers of patches in each plot were 21 for dSlo and dSlo + dSLIP1, 18 for dSlo + dSLIP1 + dSlo-C665-1164, and 15 for hSlo, hSlo + dSLIP1 and for hSlo + dSLIP1 + dSlo-C665-1164. Also examined were 11 oocytes expressing Shaker or Shaker + dSLIP1 and 6 oocytes expressing Shaker + dSLIP1 + dSlo-C665-1164. neurons. dSLIP1 mRNA is not expressed, however, in peripheral cell types that express dSlo mRNA. Northern blot analysis with a dSLIP1 probe detected a single band of $3.6 \mathrm{~kb}$ in whole-embryo mRNA (Fig. 2, right). The genomic location of the dSLIP1 gene was determined by probing polytene chromosome squashes with dSLIP1 sequences. Cytological examination unambiguously identified the dSLIP1 gene as residing on the fourth chromosome between bands $102 \mathrm{C}-\mathrm{D}$ (data not shown). This region does not contain a high density of genetic markers, and no known mutations in this area appear to involve the dSLIP1 gene (FlyBase).

\section{Domains mediating the interaction between dSlo and dSLIP1}

The interaction between the C-terminal domain of dSlo and dSLIP1 was examined in vitro. Each protein was expressed in bacteria and isolated as either a GST or pHis fusion protein. Pull-down experiments were performed by binding either GSTdSlo or GST-dSLIP1 proteins to a glutathione-agarose column and passing the other protein as a polyHis fusion over the column. Bound proteins were eluted by applying reduced glutathione, separated by polyacrylamide gel electrophoresis, and visualized by Western blot analyses. The results show that GSTdSLIP1 specifically retained pHis-dSlo-C665-1164 (Fig. 3A, left). The region of dSlo that mediates the interaction with dSLIP1 was defined by similar experiments in which different C-terminal domain fragments of dSlo were produced as GST fusion proteins and bound to glutathione-agarose beads before the application of pHis-dSLIP1 protein. The results demonstrate that inclusion of the region of dSlo between amino acids 1032 and 1164 retained dSLIP1 protein, whereas further deletion abolished the interaction (Fig. 3A, right). Similar results were obtained by complementary two-hybrid analyses. To localize more precisely the domain of dSLIP1 that mediates the interaction with dSlo, we performed two-hybrid experiments with dSlo-C665-1164 and different regions of dSLIP1. These experiments localized the interacting domain to the C-terminal 100 residues of dSLIP1; further deletions from either end resulted in loss of the interaction (Tables 1, 2).

\section{dSlo antibody coimmunoprecipitates dSLIP1}

The pull-down experiments indicated a stable interaction in vitro between dSlo-C665-1164 and dSLIP1. Therefore, dSlo and dSLIP1 were transiently cotransfected into COS-7 cells, and total cellular proteins were used for immunoprecipitations with antibodies directed against either dSlo, dSLIP1, or FLAG (control). Immunoprecipitated proteins were prepared as a Western blot and probed with dSLIP1 antibodies. Figure $3 B$ shows that immunoprecipitations with dSlo antibodies coprecipitated dSLIP1.

\section{dSLIP1 decreases dSlo current amplitudes}

Functional interactions between dSlo and dSLIP1 were examined in inside-out patches from Xenopus oocytes expressing either dSlo alone or dSlo and dSLIP1. Before injection, in vitro synthesized dSLIP and dSlo-C665-1164 mRNAs were adjusted to equal approximately molar ratios on the basis of spectrophotometric and agarose gel analyses, while dSlo mRNA was injected at threeto fivefold lower concentrations. Current amplitudes were measured at $100 \mathrm{mV}$ in the presence of $10 \mu \mathrm{M}$ internal $\mathrm{Ca}^{2+}$, using electrodes of similar resistances $(\sim 3 \mathrm{M} \Omega)$. In a representative experiment (Fig. $4 A$ ), patches from dSlo-expressing oocytes had current amplitudes of $3.0 \pm 2.5 \mathrm{nA}(n=21)$, whereas similar patches from oocytes coexpressing dSlo and dSLIP1 showed current amplitudes of $0.8 \pm 1.9 \mathrm{nA}(n=21 ; p<0.005)$. The 
A

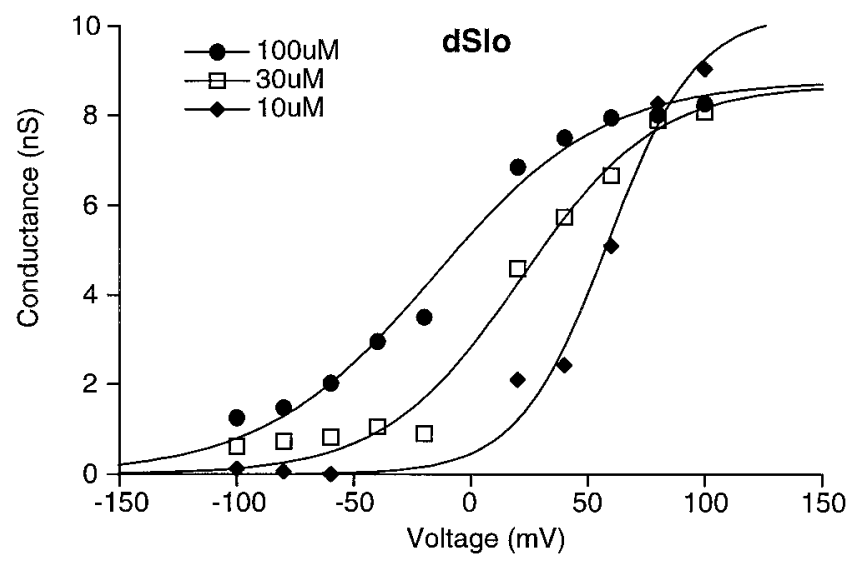

B

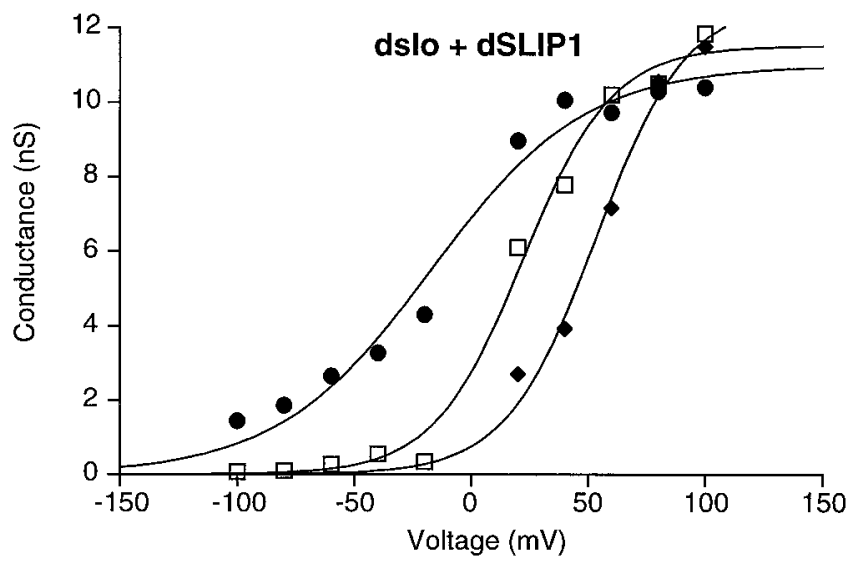

Figure 5. dSLIP1 does not change the calcium dependence of dSlo. Excised patches from oocytes expressing dSlo $(A)$, dSlo and dSLIP1 $(B)$, or dSlo, dSLIP1, and dSlo-C665-1164 were exposed to 10, 30, and $100 \mu \mathrm{M}$ free $\mathrm{Ca}^{2+}$. Patch conductance was determined by dividing the steady-state current during a $500 \mathrm{msec}$ voltage step by the holding potential. Data were fit to a Boltzmann equation of the form $I=I_{\max } /\left(1+\exp k \cdot\left(V-V_{0.5}\right)\right)$. $C$, The resulting potentials of half-activation $\left(V_{0.5}\right)$ were averaged for three to five patches and plotted as a function of $\mathrm{Ca}^{2+}$ concentration; error bars represent SE.

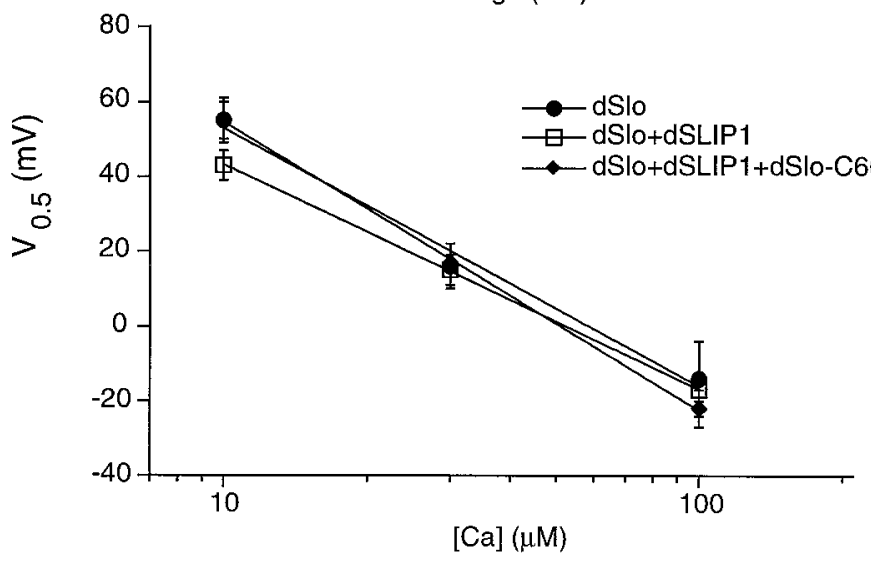

reduction in patch current was specific for the interaction between dSlo and dSLIP1 because the effect was reversed by coexpression with dSlo-C665-1164, the fragment used to screen for dSLIP1. In the experiment shown, current amplitudes in patches from oocytes expressing dSlo, dSLIP1, and dSlo-C665-1164 were no different from those for dSlo alone, $3.6 \pm 1.9 \mathrm{nA}(n=18 ; p>$ $0.1)$. dSlo current reduction by coexpression with dSLIP1 gave similar results in 12 of 15 experiments that used different batches of oocytes; the average reduction was $71 \pm 18 \%$. In seven batches of oocytes, inhibition of this effect by dSlo-C665-1164 was examined; the current was rescued at least partially in six batches, resulting in a $3.4 \pm 1.1$-fold increase in the average patch current as compared with oocytes expressing only dSlo and dSLIP1. There was no effect of dSlo-C665-1164 on dSlo current amplitudes $(1.7 \pm 0.8 \mathrm{nA}, n=9$ vs $2.0 \pm 1.2, n=11 ; p>0.5)$. In three of five experiments in which the human BK channel, hSlo, was

substituted for dSlo, the same specific inhibitory effect of dSLIP1 was observed (Fig. 4B). On average, the current seen in oocytes expressing hSlo and dSLIP1 was reduced by $69 \pm 10 \%$ as compared with oocytes expressing only hSlo. Oocytes expressing hSlo, dSLIP1, and dSlo-C665-1164 displayed a $2.4 \pm$ 1.1-fold increase in current as compared with oocytes expressing hSlo and dSLIP1. In contrast, dSLIP1 did not reduce currents in oocytes expressing Shaker channels (Fig. 4C). The effect of dSLIP1 on Slo currents was sensitive to the amount of Slo mRNA injected. When Slo mRNA was injected in excess to dSLIP1, the reduction in current amplitude was reduced or eliminated.

To investigate whether the reduction of patch current at a given voltage and calcium concentration was attributable to a reduced calcium sensitivity of dSlo channels in the presence of dSLIP1, we measured the patch conductance holding at -100 to $+100 \mathrm{mV}$ in three concentrations of intracellular $\mathrm{Ca}^{2+}$ for patches expressing 


\section{A}

hSlo
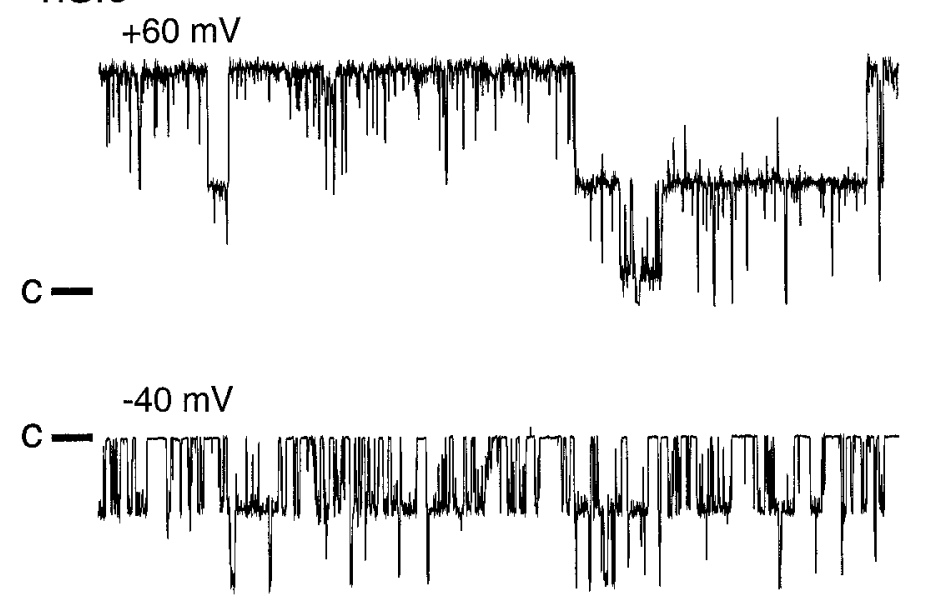

B

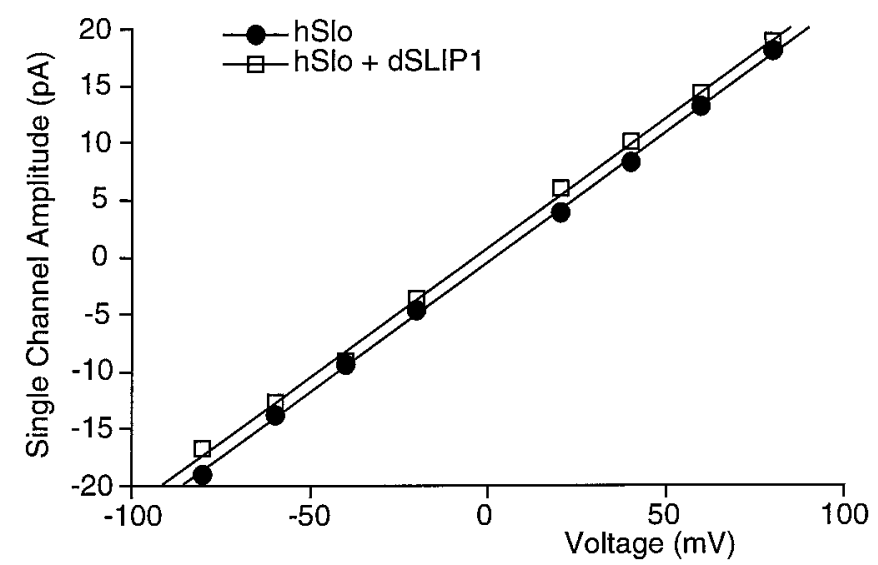

hSlo + dSLIP1
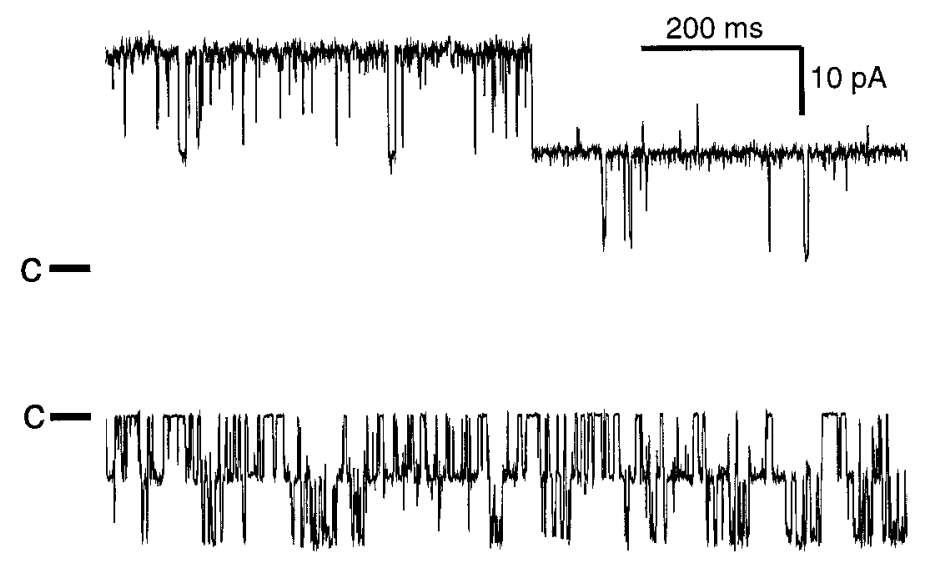

C

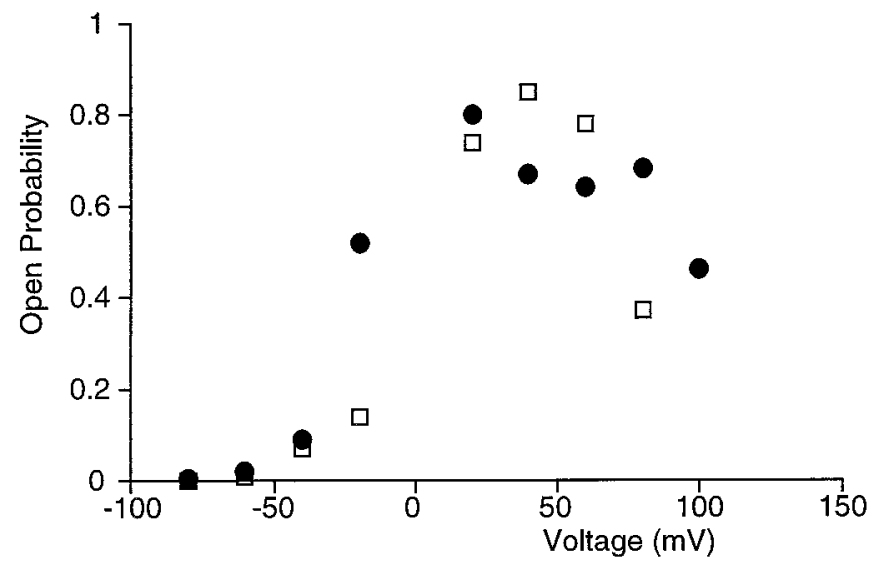

Figure 6. Coexpression with dSLIP1 does not affect the single-channel conductance or the open probability of hSlo channels. $A$, Traces (1 sec) of steady-state recordings from patches excised from an oocyte expressing hSlo (left) or hSlo and dSLIP1 (right). Patches were exposed to $100 \mu \mathrm{M}$ free Ca ${ }^{2+}$ and held at $+60 \mathrm{mV}$ (top) or $-40 \mathrm{mV}$ (bottom). Both patches contained two channels; the closed levels are indicated on the left. B, Single-channel amplitudes as a function of voltage in two patches containing two channels ( filled circles, same patch as $A$ ) or a single-channel (open squares) from an oocyte expressing hSlo or hSlo and dSLIP1, respectively. Amplitudes were determined by fitting gaussian distributions to amplitude histograms representing at least 1000 events. Linear regression analysis yielded single-channel conductances of $227 \mathrm{pS}$ for hSlo and $226 \mathrm{pS}$ for hSlo and dSLIP1. $C$, Single-channel open probability as a function of voltage for the two patches in $B$.

dSlo alone (Fig. $5 A$ ) or together with dSLIP1 (Fig. $5 B$ ). Data were fit to a Boltzmann relationship, and the resulting values for the voltage of half-activation $\left(V_{0.5}\right)$ were averaged for three to five patches and plotted as a function of $\mathrm{Ca}^{2+}$ concentration in Figure $5 C$; no significant differences were observed at any of the $\mathrm{Ca}^{2+}$ concentrations that were examined.

To investigate further the mechanism underlying the reduction in Slo currents mediated by dSLIP1, we examined single hSlo channels. Channels from oocytes injected either with hSlo alone or with hSlo and dSLIP1 were exposed to $100 \mu \mathrm{M} \mathrm{Ca}{ }^{2+}$ and showed the same single-channel amplitude as a function of voltage (Fig. 6A,B). The average single-channel conductance was $242 \pm 16 \mathrm{pS}$ in three patches with hSlo alone and $224 \pm 5 \mathrm{pS}$ in four patches with hSlo and dSLIP1 $(p>0.05)$. The open probability $\left(P_{\mathrm{o}}\right)$ as a function of voltage also was not affected by coexpression with dSLIP1 (Fig. 6C), further confirming that dSLIP1 did not affect the calcium sensitivity of hSlo channels.

Because the gating and conduction properties of Slo channels were not obviously affected by dSLIP1, the reduction in macroscopic current amplitudes probably resulted from a reduced number of channels in the plasma membrane. This possibility was examined further by immunocytochemistry with dSlo antiserum on COS-7 cells that had been transfected with dSlo alone or together with dSLIP1. In cells expressing only dSlo, a reticular punctate staining pattern was obtained, with dSlo immunoreactivity clearly seen on the outer membranes (Fig. 7, left). In contrast, cotransfected cells showed diffuse internal staining with little, if any, dSlo immunoreactivity on the cell surface (Fig. 7, right).

\section{DISCUSSION}

The results presented here demonstrate that dSLIP1 selectively interacts with the C-terminal domain of BK channels. When coexpressed in Xenopus oocytes, dSLIP1 reduces macroscopic BK currents as compared with expression of BK channels alone, without affecting unit current amplitude $(I)$ or open probability $\left(P_{\mathrm{o}}\right)$. Taken together with immunocytochemical results from co- 
Figure 7. Immunostaining of COS-7 cells. COS-7 cells transiently transfected with dSlo (left) or cotransfected with dSlo and dSLIP1 (right) were immunostained with dSlo antiserum. Cells expressing dSlo show only a reticular punctate staining pattern with clear cell surface staining, whereas cells transfected with dSlo and dSLIP1 show a diffuse intracellular staining pattern.
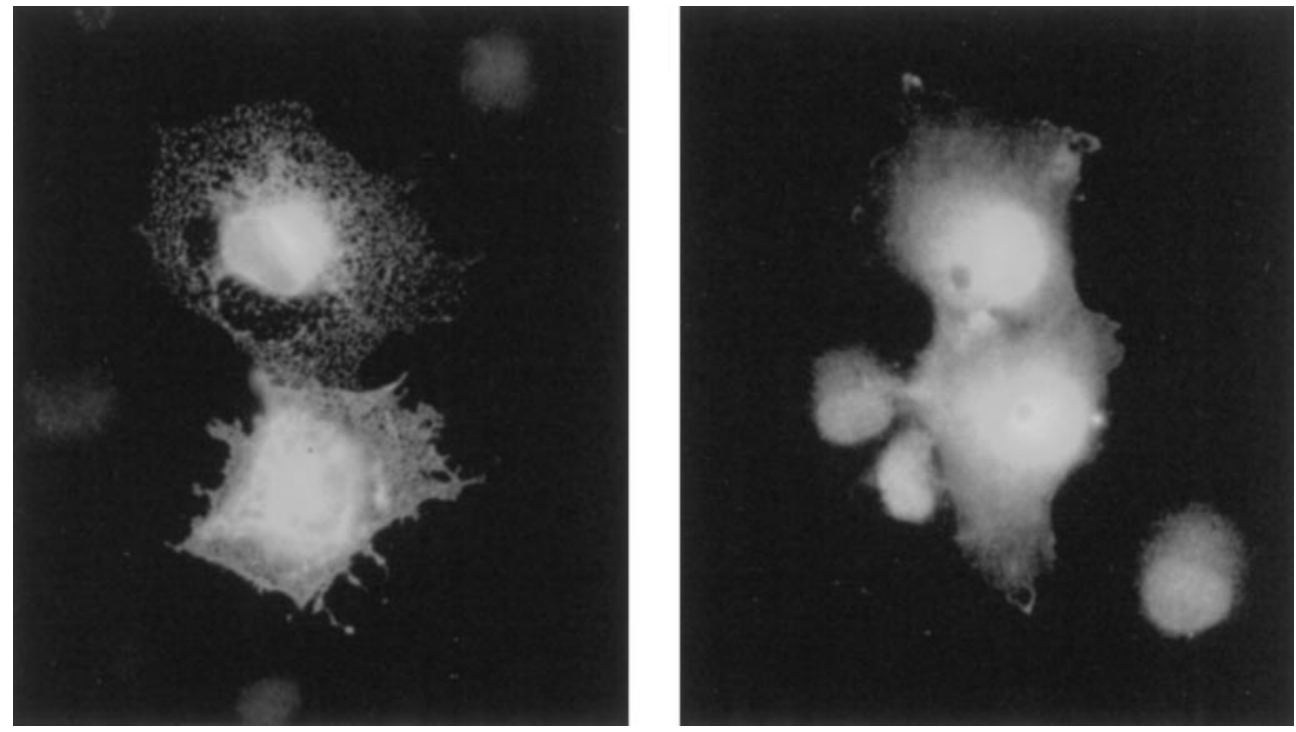

transfected COS-7 cells, it is likely that dSLIP1 limits the number of functional BK channels in the plasma membrane.

Mechanisms for regulating channel density will have significant effects on neurons and other excitable cells. For neuronal sodium channels, intracellular pools of the $\alpha$-subunit appear before the emergence of functional sodium channels on the cell surface, and it is likely that maturation, translocation, and plasmalemmal insertion are accompanied by association with $\beta$-subunits (Scheinman, 1989; Sutkowski and Catterall, 1990; Isom et al., 1995). A similar mechanism may operate in cardiac myocytes $(\mathrm{Qu}$ et al., 1995). Coexpression of calcium channel $\alpha$ - and $\beta$-subunits increases the amplitudes of the currents and the number of highaffinity drug and toxin-binding sites as compared with expression of $\alpha$-subunits alone (Mori et al., 1991; Williams et al., 1992; Stea, 1993). Voltage-gated potassium channels may associate with one or more $\beta$-subunits. The $\beta 2$ subunit acts as a chaperone, regulating channel density and current amplitude by increasing the number of channels in the plasma membrane (Rettig et al., 1994; Shi et al., 1996).

For BK channels, post-translational regulation of current density is well documented in developing parasympathetic neurons such as chick ciliary ganglia (CG). In developing CG, or in cultured $\mathrm{CG}$ in the absence of target tissue, BK channel mRNA is present, but no BK currents are detected. However, when CG neurons are cultured in the presence of target tissue extract, BK channel activity appears, and this regulation is independent of protein synthesis (Subramony et al., 1996; Subramony and Dryer, 1997). Although the underlying mechanisms have not yet been elucidated, the results are consistent with a regulated interaction, in the absence of target tissue, between intracellular BK channels and a dSLIP1 homolog.

These studies implicate post-translational mechanisms for the regulated appearance of functional channels in the plasma membrane. dSLIP1 may represent a component of such a system, retaining BK channel $\alpha$-subunits in an intracellular pool until a physiological cue induces translocation into the plasma membrane. In our studies the effects of dSLIP1 may be overcome by the additional expression of $\mathrm{BK} \alpha$-subunits, suggesting the possibility that one molecule of dSlo binds to several (four?) molecules of dSLIP1.

The C-terminal domain of dSLIP1 is required for the interac- tions with $\mathrm{BK} \alpha$-subunits, whereas the $\mathrm{N}$-terminal domain of dSLIP1 has limited homology with the PDZ domain of $\alpha 1$ syntrophin, which has been shown to bind nitric oxide synthase and has been implicated in calcium binding (Brenman et al., 1996; Newbell et al., 1997). Similarly, this domain of dSLIP1 may mediate interactions with additional regulatory components, perhaps in a calcium-dependent manner.

\section{REFERENCES}

Adams ME, Dwyer TM, Dowler LL, White RA, Froehner SC (1995) Mouse alpha 1- and beta 2-syntrophin gene structure, chromosome localization, and homology with a discs large domain. J Biol Chem 270:25859-25865.

Adelman JP, Shen KZ, Kavanaugh MP, Warren RA, Wu YN, Lagrutta A, Bond CT, North RA (1992) Calcium-activated potassium channels expressed from cloned complementary DNAs. Neuron 9:209-216.

Atkinson NS, Robertson GA, Ganetzky B (1991) A component of calcium-activated potassium channels encoded by the Drosophila slo locus. Science 253:551-555.

Bond CT, Pessia M, Xia XM, Lagrutta A, Kavanaugh MP, Adelman JP (1994) Cloning and expression of a family of inward rectifier potassium channels. Receptors Channels 2:183-191.

Brenman JE, Chao DS, Gee SH, McGee AW, Craven SE, Santillano DR, Wu Z, Huang F, Xia H, Peters MF, Froehner SC, Bredt DS (1996) Interaction of nitric oxide synthase with the postsynaptic density protein PSD-95 and $\alpha 1$-syntrophin mediated by PDZ domains. Cell 84:757-767.

Butler A, Tsunoda S, McCobb DP, Wei A, Salkoff L (1993) mSlo, a complex mouse gene encoding "maxi" calcium-activated potassium channels. Science 261:221-224.

Chung SK, Reinhart PH, Martin BL, Brautigan D, Levitan IB (1991) Protein kinase activity closely associated with a reconstituted calciumactivated potassium channel. Science 253:560-562.

Cole WC, Sanders KM (1989) G-proteins mediate suppression of $\mathrm{Ca}^{2+}$. activated $\mathrm{K}$ current by acetylcholine in smooth muscle cells. Am J Physiol 257:C596-C600.

Dawson RMC, Elliot DC, Elliot WH, Jones UM (1969) Data for biochemical research. New York: Oxford UP.

Dworetzky SI, Boissard CG, Lum-Ragan JT, McKay MC, Post-Munson DJ, Trojnacki JT, Chang CP, Gribkoff VK (1996) Phenotypic alteration of a human BK (hSlo) channel by hSlo $\beta$-subunit coexpression: changes in blocker sensitivity, activation/relaxation and inactivation kinetics, and protein kinase A modulation. J Neurosci 16:4543-4550.

Ewald DA, Williams A, Levitan IB (1985) Modulation of single $\mathrm{Ca}^{2+}$. dependent $\mathrm{K}^{+}$channel activity by protein phosphorylation. Nature 315:503-506.

Gibson J, Hyvonen M, Musacchio A, Saraste M, Birney E (1994) PH domain: the first anniversary. Trends Biochem Sci 19:349-353. 
Gola M, Crest M (1993) Colocalization of active KCa channels and $\mathrm{Ca}^{2+}$ channels within $\mathrm{Ca}^{2+}$ domains in Helix neurons. Neuron 10:689-699.

Gyuris J, Golemis E, Chertkov H, Brent R (1993) Cdi1, a human G1 and S-phase protein phosphatase that associates with Cdk2. Cell 75:791-803.

Hanner M, Schmalhofer WA, Munujos P, Knaus HG, Kaczorowski GJ, Garcia ML (1997) The beta subunit of the high-conductance calciumactivated potassium channel contributes to the high-affinity receptor for charybdotoxin. Proc Natl Acad Sci USA 94:2853-2858.

Hotson JR, Prince DA (1980) A calcium-activated hyperpolarization follows repetitive firing in hippocampal neurons. J Neurophysiol 43:409-419.

Isom LL, Ragsdale SS, DeJongh KS, Westenbroek RE, Reber BFX, Scheuer T, Catterall WA (1995) Structure and function of the $\beta 2$ subunit of brain sodium channels, a transmembrane glycoprotein with a CAM motif. Cell 83:433-442.

Issa NP, Hudspeth AJ (1994) Clustering of $\mathrm{Ca}^{2+}$ channels and $\mathrm{Ca}^{2+}$ activated $\mathrm{K}^{+}$channels at fluorescently labeled presynaptic active zones of hair cells. Proc Natl Acad Sci USA 91:7578-7582.

Köhler M, Hirschberg B, Bond CT, Marrion NV, Kinzie JM, Maylie J, Adelman JP (1996) Small-conductance calcium-activated potassium channels from mammalian brain. Science 273:1709-1714.

Krapivinsky G, Gordon EA, Wickman K, Velimirovic B, Krapivinsky L, Clapham DE (1995a) The G-protein-gated atrial $\mathrm{K}^{+}$channel $\mathrm{I}_{\mathrm{KACh}}$ is a heteromultimer of two inwardly rectifying $\mathrm{K}^{+}$channel proteins. Nature 374:135-141.

Krapivinsky G, Krapivinsky L, Velimirovic B, Navaroo KW B, Clapham DE (1995b) The cardiac inward rectifier $\mathrm{K}^{+}$channel subunit, CIR, does not comprise the ATP-sensitive $\mathrm{K}^{+}$channel, $\mathrm{I}_{\mathrm{KATP}}$. J Biol Chem 270:28777-28779.

Lancaster B, Nicoll R (1987) Properties of two calcium-activated hyperpolarizations in rat hippocampal neurones. J Physiol (Lond) 389:187-203.

Ma J, Ptashne M (1988) Converting a eukaryotic transcriptional inhibitor into an activator. Cell 55:443-446.

Madison DV, Nicoll RA (1984) Control of the repetitive discharge of rat CA1 pyramidal neurons in vitro. J Physiol (Lond) 354:319-331.

McManus OB, Helms LM, Pallanck L, Ganetzky B, Swanson R, Leonard RJ (1995) Functional role of the beta subunit of high conductance calcium-activated potassium channels. Neuron 14:645-650.

Mori Y, Friedrich T, Kim M-S, Mikami A, Nakai J, Ruth P, Bosse E, Hofmann F, Flockerzi V, Furuichi T, Mikoshiba K, Imoto K, Tanabe T, Numa S (1991) Primary structure and functional expression from complementary DNA of a brain calcium channel. Nature 350:398-402.

Newbell BJ, Anderson JT, Jarrett HW (1997) $\mathrm{Ca}^{2+}$-calmodulin binding to $\alpha 1$-syntrophin: syntrophin is also a $\mathrm{Ca}^{2+}$-binding protein. Biochemistry 36:1295-1305.

O'Neil J, Bier E (1994) Double-label in situ hybridization using biotin and digoxigenin-tagged RNA probes. Biotechniques 17:870-875.

Qu Y, Isom LL, Westenbroek RE, Rogers JC, Tanada TN, McCormick
KA, Scheuer T, Catterall WA (1995) Modulation of cardiac $\mathrm{Na}^{+}$ channel expression in Xenopus oocytes by $\beta 1$ subunits. J Biol Chem 270:25696-25701.

Quan F, Wolfgang W, Forte M (1993) A Drosophila G-protein $\alpha$ subunit, $\mathrm{G}_{\mathrm{f} \alpha}$, expressed in a spatially and temporally restricted pattern during Drosophila development. Proc Natl Acad Sci USA 90:4236-4240.

Reinhart PH, Chung S, Martin BL, Brautigan DL, Levitan IB (1991) Modulation of calcium-activated potassium channels from rat brain by protein kinase A and phosphatase 2A. J Neurosci 11:1627-1635.

Rettig J, Heinemann SH, Wunder F, Lorra C, Parcej DN, Dolly JO, Pongs O (1994) Inactivation properties of voltage-gated $\mathrm{K}^{+}$channels altered by presence of $\beta$-subunit. Nature 369:289-294.

Robitaille R, Garcia ML, Kaczorowski GJ, Charlton MP (1993) Functional colocalization of calcium and calcium-gated potassium channels in control of transmitter release. Neuron 11:645-655.

Scheinman RI, Auld VJ, Goldin AL, Davidson N, Dunn RJ, Catterall WA (1989) Developmental regulation of sodium channel expression in the rat forebrain. J Biol Chem 264:10660-10666.

Schiestl RH, Gietz RD (1989) High efficiency transformation of intact yeast cells using single stranded nucleic acids as a carrier. Curr Genet 16:339-346.

Scornik FS, Codina J, Birnbaumer L, Stefani E, Toro L (1992) Activation of calcium-activated potassium (KCa) channels from pig coronary artery by GTP $\gamma \mathrm{S}$ may involve a G-protein. Biophys J 61:A254.

Shi G, Nakahira K, Hammond S, Rhodes KJ, Schechter LE, Trimmer JS (1996) $\beta$-Subunits promote $\mathrm{K}^{+}$channel surface expression through effects early in biosynthesis. Neuron 16:843-852.

Stea A, Dubel SJ, Pragnell M, Leonard JP, Campbell KP, Snutch TP (1993) A beta subunit normalizes the electrophysiological properties of a cloned N-type calcium channel. Neuropharmacology 32:1103-1116.

Storm JF (1987) Action potential repolarization and a fast afterhyperpolarization in rat hippocampal pyramidal cells. J Physiol (Lond) 385:733-759.

Subramony P, Dryer SE (1997) Neuregulins stimulate the functional expression of $\mathrm{Ca}^{2+}$-activated $\mathrm{K}^{+}$channels in developing chicken parasympathetic neurons. Proc Natl Acad Sci USA 94:5934-5938.

Subramony P, Raucher S, Dryer L, Dryer SE (1996) Posttranslational regulation of $\mathrm{Ca}^{2+}$-activated $\mathrm{K}^{+}$currents by a target-derived factor in developing parasympathetic neurons. Neuron 17:115-124.

Sutkowski EM, Catterall WA (1990) $\beta 1$ subunits of sodium channels. J Biol Chem 265:12393-12399.

Toro L, Ramos-Franco J, Stefani E (1990) GTP-dependent regulation of myometrial $\mathrm{KCa}$ channels incorporated into bilayers. J Gen Physiol 96:373-394.

Williams ME, Feldman DH, McCue AF, Brenner R, Velicelebi G, Ellis SB, Harpold MM (1992) Structure and functional expression of $\alpha 1$, $\alpha 2$, and $\beta$ subunits of a novel human neuronal calcium channel subtype. Neuron 8:71-84.

Yarom Y, Sugimori M, Llinás R (1985) Ionic currents and firing patterns of mammalian vagal motoneurons in vitro. Neuroscience 16:719-737. 